\title{
Optimized Sample Preparation for the Imaging of Silicon Defects using Plan View Transmission Electron Microscopy
}

\author{
Nathan Wang*, Alline Myers, Max Sidorov, Heather Koo, Caiwen Yuan, Bryan Tracy, Susan Li \\ Spansion Inc., Sunnyvale, CA, USA
}

Plan view TEM (PTEM), with its top down perspective, is a powerful technique to observe silicon related defects, such as dislocations, stacking faults and gate oxide ruptures. Besides direct defect identification, PTEM can be effectively used to locate a defect when its precise location is not available. Once the defect is found using the large area available in plan view specimens, then a cross-section TEM (XTEM) can be performed to get detailed layer information. Such so called "3D TEM" has been applied more and more in modern device failure analysis [1]. However, sometimes, we are not able to see a defect in a PTEM image even if it is in the field of view. The following case is an example. Analysis of such a case will help improve the "hit rate" for device failure analysis.

Two flash memory devices were received for analysis and both samples failed erase. PTEM was performed for both samples and the results are shown in Figures 1 and 2. Figure 1 is for the sample \#1 and clearly shows the oval shape of the defect. Cross sectioning along the short axis of the oval (perpendicular to poly line) was performed and the result is shown in Figure 3A. XTEM indicates that the defect is a microcrack in the silicon substrate. The higher magnification images are shown in Figures 4A. However, the PTEM image in Figure 2 does give any clue about the defect at the target location. A cross-section was also performed on this sample. The cut is parallel to the known bad poly line. Fortunately, the defect was caught by XTEM and is shown in Figures 3B and 4B. It should be a very similar defect as that in Figure 1 because it has the same length and depth as the first sample. However, the silicon defect is nearly invisible in this image.

It is worthwhile to compare the two samples in order to figure out why the PTEM image does not show a defect in Figure 2 as clearly as in Figure 1. The XTEM images for both samples are listed side by side in same magnification in Figure 3. Layer structure and sample thickness for both samples are comparable easily. Sample \#1 has very thick Si substrate with a very thin polysilicon top layer. Sample \#2 has thin substrate but a thick poly layer capped with CoSi. PTEM samples commonly have a sandwich structure that consists of polysilicon, gate oxide/ or ONO and silicon substrate layers. The contrast in a PTEM image is a combination from each of these individual layers, superimposed. Since ONO is a uniform, non-crystalline and thin, the important layers in contrast formation would be the $\mathrm{Si}$ substrate, Polysilicon and CoSi thin films. The substrate is single crystal and its contribution to total contrast is mainly mass-thickness if the sample is tilt off the zone axis. The polysilicon and the CoSi layers are the most likely candidates to interfere with the contrast of the silicon substrate defect. In our case, the defect is a silicon microcrack that is the same material as polysilicon. It was deduced that the contrast from the defect was not buried in the contrast from poly grains because local strain around the defect. It is also noticed that CoSi layer on top of poly has been removed in the sample \#1 but only partially removed in the sample \#2. This layer is very critical in forming TEM contrast. In Figure 1, very weak contrast is shown at center area. Strong contrast is shown at side strip (marked as thick area) because the CoSi is still there. The contrast at this area is quite similar as the one in Figure 2. Cobalt is a heavy transition metal and it has higher $\mathrm{Z}$ than Silicon. The higher $\mathrm{Z}$ material scatters more than the 
lower $\mathrm{Z}$ material and results much stronger contrast than lower $\mathrm{Z}$ material [2]. It is now clear that $\mathrm{CoSi}$ layer blocks the contrast that formed from defect in the silicon substrate.

The orientation and thickness of the silicon substrate is important during defect imaging. When the orientation is off the $<110>$ zone, the Si contribution to total contrast is only mass thickness contrast. As such, it just slightly blurs the image. Thick samples on the other hand are less subject to bend contours. The fringes from bend contours may interfere with the successful imaging of substrate defects.

According to above discussion, we can draw some conclusions regarding the optimized preparation of plan view TEM specimens:

- It is necessary to remove any metal silicide layers in order to preserve the relatively weaker diffraction contrast from silicon substrate defects

- Silicon substrate thickness is not very important in helping to locate defects, although a thinner sample may provide better resolution. Silicon defects can be readily found with thick samples because the penetration of the electron beam in quite large especially at $300 \mathrm{kV}$. Very thin TEM samples are not necessary to locate silicon defects and may actually be less suitable for failure analysis.

\section{Reference}

[1] N. Wang and Sabbas Daniel, Microelectronic Failure Analysis Desk Reference 2002 suppl., ASM international, Materials Park, OH, (2002), pp.21-28

[2] D. A. Williams and C. B. Carter, Transmission Electron Microscopy, Plenum Press, (New York, 1996), p.98.

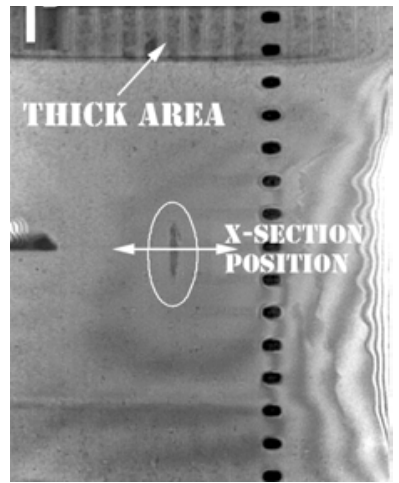

Fig. 1.PTEM image for the sample \#1;

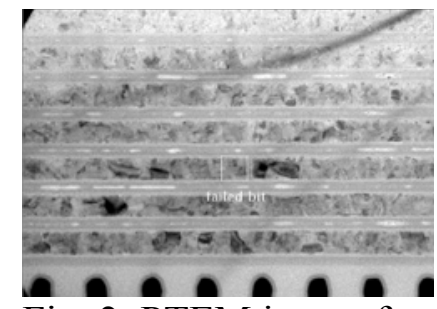

Fig. 2. PTEM image for Sample \#2;
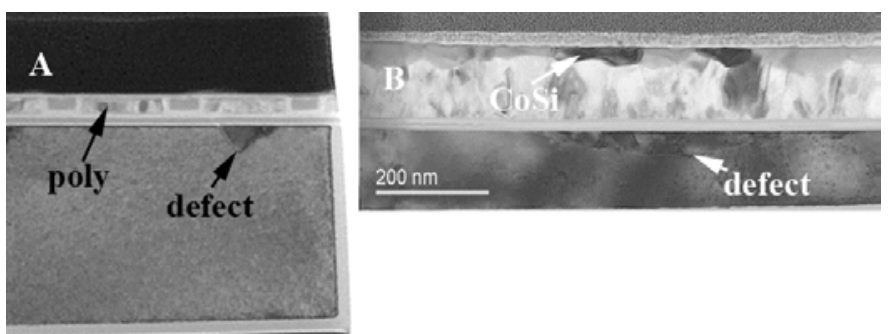

Fig. 3. XTEM image for the sample \#1 (A) and 2(B)

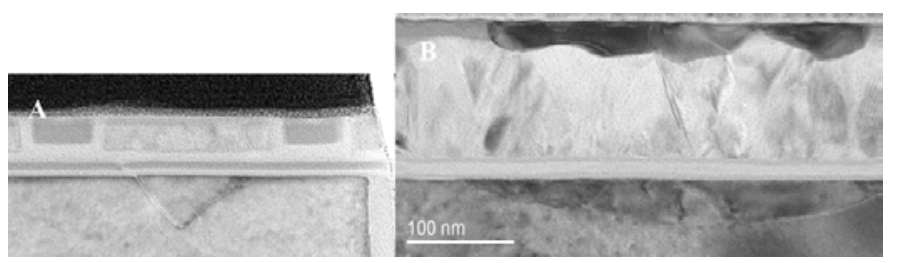

Fig. 4. Zoom-in XTEM image from Fig. 3 